\section{IMPACTO DA CONSULTA FARMACÊUTICA EM PACIENTES POLIMEDICADOS COM ALTO RISCO CARDIOVASCULAR}

Walleri Christini Torelli Reis ${ }^{1}$

Camila dos Santos Bernardo ${ }^{2}$ Thais Teles de Souza ${ }^{3}$ Aline De Fátima Bonetti ${ }^{3}$ Maria Luiza Drechsel Favero ${ }^{4}$ Vânia Mari Salvi Andrzejevski ${ }^{5}$

\section{RESUMO}

Introdução: A ascensão dos serviços clínicos farmacêuticos possibilita que este profissional realize ações voltadas ao paciente, promovendo o uso racional de medicamentos e a melhoria da qualidade de vida. Estas ações podem ser realizadas a partir de consultas farmacêuticas, com a finalidade de otimizar a farmacoterapia do paciente e acompanhar periodicamente as suas condições de saúde a fim de avaliar a eficácia do tratamento. Objetivo: avaliar do impacto do serviço farmacêutico em pacientes polimedicados atendidos em ambulatório de hospital de grande porte. Métodos: foram coletados os dados obtidos das consultas realizadas durante seis meses no Ambulatório de Atenção Farmacêutica e estes dados foram analisados estatisticamente. Resultados: houve um aumento significativo do controle das morbidades entre a primeira e a segunda consulta, de 2,52 ( $\pm 1,465)$, em média, para 2,95 $( \pm 1,519)$, $p=0.003$, indicando potencial benefício do cuidado farmacêutico sobre a saúde do paciente. Também foi observada uma constância na quantidade de queixas e no número de intervenções realizadas, reiterando a necessidade de acompanhamento continuado para a resolução de todos os problemas do paciente. Conclusões: o acompanhamento ambulatorial por farmacêutico clínico apresenta potencial para a obtenção de desfechos positivos na saúde do paciente.

Palavras-chaves: Farmácia clínica. Polifarmácia. Cuidado farmacêutico.

\section{INTRODUÇÃO}

Os serviços farmacêuticos clínicos representam ações cognitivas do farmacêutico, direcionadas ao paciente, profissionais de saúde e comunidade, voltadas principalmente ao rastreamento de doenças, manejo de problemas autolimitados, educação em saúde, revisão farmacoterapia, gestão da doença e acompanhamento farmacoterapêutico. Esses serviços destinam-se a otimizar a farmacoterapia, melhorando assim desfechos clínicos, humanísticos e econômicos. ${ }^{1-4}$.

Apesar do termo "farmácia clínica" existir desde a década de 1960, no Brasil esta ideia ainda se encontra em desenvolvimento. A política de atenção farmacêutica foi discutida pela primeira vez no Encontro Nacional de Assistência Farmacêutica e Política de Medicamentos em 1988, porém apenas em 2002, no Consenso Brasileiro de Atenção Farmacêutica esta prática foi melhor estabelecida no país ${ }^{5,6}$.

Considerando o contexto mundial, inúmeras publicações têm demonstrado o impacto dos serviços farmacêuticos clínicos em diferentes populações e ambientes de cuidado ${ }^{7-11}$. Uma revisão sistemática publicada recentemente apontou reduções significativas nos valores de pressão arterial, colesterol total e índice de massa corporal em pacientes que receberam intervenções do farmacêutico clínico ${ }^{8}$. Paralelamente, uma overview de revisões sistemáticas, publicada em 2015, apontou benefícios significativos dos serviços farmacêuticos clínicos em pacientes com hipertensão arterial sistêmica e diabetes ${ }^{9}$.
Evidências recentes apontam que os serviços farmacêuticos clínicos tendem a ser mais efetivos em pacientes que apresentam maior tendência para a ocorrência de problemas da farmacoterapia ${ }^{12-15}$. Dentre esses fatores, destacam-se o diagnóstico de múltiplas comorbidades, a polifarmácia e a presença de limitações para a gestão da farmacoterapia, sejam elas físicas ou cognitivas ${ }^{16,17}$.

Estas situações podem ser atribuídas à atual transição demográfica e epidemiológica em que se encontram o Brasil e o mundo, contribuindo para a maior prevalência de doenças crônicas, que exigem tratamentos combinados, tais como hipertensão arterial sistêmica e diabetes ${ }^{18,19}$. O uso concomitante de diversos medicamentos, apesar de muitas vezes ser necessário e inevitável, está relacionado ao aumento de reações adversas, interações medicamentosas, intoxicações, danos físicos e psicológicos ${ }^{20,21}$.

Serviçosfarmacêuticos ambulatoriais têmganhado ascensão à medida que se identifica a sua necessidade iminente. Dados da Organização Mundial de Saúde indicam que as taxas de não adesão ao tratamento de doenças crônicas podem ultrapassar $50 \%{ }^{22}$. No Brasil, um levantamento realizado pelo Ministério da Saúde, através da implantação de consultas farmacêuticas no Sistema Único de Saúde, indicou que mais de 90\% dos pacientes apresentavam algum problema de adesão ${ }^{23}$.

A consulta farmacêutica representa uma atividade complexa, que incorpora diferentes serviços farmacêuticos clínicos, de acordo com as necessidades do paciente, a fim de melhorar sua saúde e qualidade de vida ${ }^{1,2}$. Apesar do benefício
1 Departamento de Farmácia, Universidade Federal da Paraíba 2 Universidade Federal do Paraná 3 Pós-Graduação em Ciências Farmacêuticas, Universidade Federal Do Paraná 4 Departamento de Farmácia, Universidade Federal da Paraná 5 Complexo Hospital de Clínicas, Universidade Federal do Paraná

Recebido: 15/04/2018

Revisado: 14/04/2018

Aceito: 29/05/2018

Como citar este artigo:

Reis WCT, Bernardo CS, Souza

TT, Bonetti AF, Favero MLD e Andrzejevski VMS. Impacto da consulta farmacêutica em pacientes polimedicados com alto risco cardiovascular. Rev. Bras. Farm. Hosp. Serv. Saúde, 9(2): 1-5, 2019.

Doi: $10.30968 /$ rbfhss.2018.092.003

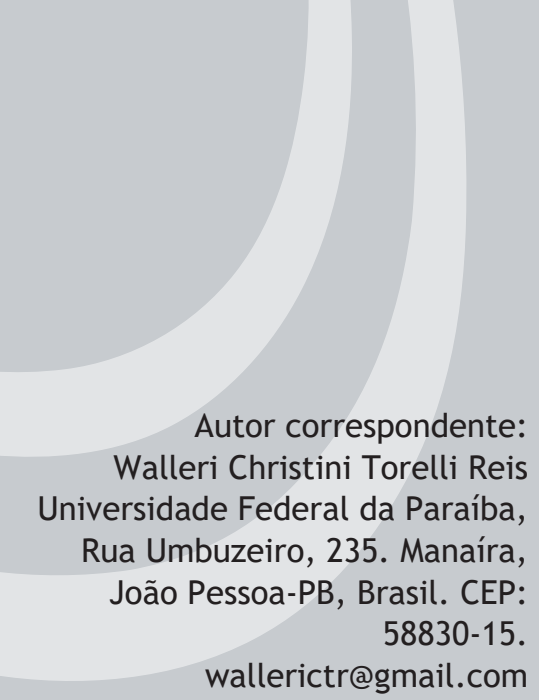


presumido desse tipo de acompanhamento, diferentemente dos países europeus e anglo-saxões, os dados nacionais sobre esta prática ainda são escassos.

Considerando tais questões, o presente estudo teve como objetivo descrever os dados provenientes de consultas realizadas em um ambulatório de Atenção Farmacêutica, sediado em serviço ambulatorial de um hospital de grande porte, além de mensurar o impacto dessas consultas para a melhoria das condições de saúde dos pacientes atendidos.

\section{MÉTODOS}

Trata-se de um estudo observacional descritivo e analítico. A coleta dos dados foi realizada de forma retrospectiva, a partir dos prontuários farmacêuticos do serviço de Atenção Farmacêutica do Complexo Hospital de Clínicas da Universidade Federal do Paraná (CHC-UFPR). Este serviço está em vigência no hospital há mais de 5 anos, o qual é destinado para o atendimento de pacientes que apresentam as seguintes características: maiores de 18 anos, previamente atendidos nas unidades médicas e ambulatórios de cardiologia do CHC-UFPR e polimedicados. Estes eram direcionados ao ambulatório por outros profissionais da saúde, por convite dos próprios farmacêuticos (busca ativa a partir da leitura de prontuários dos pacientes previamente hospitalizados nas unidades de internação de cardiologia do hospital) ou por interesse do paciente. Para a inclusão nesta pesquisa o paciente deveria apresentar as características supracitadas e manifestar sua anuência por meio da assinatura de um termo de consentimento livre e esclarecido.

Os dados avaliados foram provenientes dos registros dos prontuários farmacêuticos de consultas realizadas no referido serviço por farmacêuticos integrantes do programa de Residência Integrada Multiprofissional em Atenção Hospitalar do CHC/UFPR, área de concentração de Cardiologia. Ademais, o ambulatório conta com a colaboração de farmacêuticos voluntários com experiência clínica por mais de 3 anos, os quais atuam como preceptores, auxiliando na tomada de decisões durante as consultas. Adicionalmente, ao final de todos os atendimentos, são realizadas discussões dos casos juntamente com toda a equipe de farmacêuticos. Os prontuários são exclusivamente de uso farmacêutico, os quais foram desenvolvidos pela equipe de preceptores; entretanto, o farmacêutico responsável deve também realizar o registro do atendimento no prontuário do paciente, disponível para a visualização por todos os profissionais de saúde do hospital.

Os farmacêuticos residentes somente iniciavam o atendimento após a realização de um curso de capacitação teórico-prático de seis meses previamente implantados no serviço. As consultas farmacêuticas tinham como foco principal a promoção do uso racional dos medicamentos, o estímulo à adesão do paciente à farmacoterapia, bem como a resolução dos problemas relacionados à mesma.

Para o presente estudo foram coletadas as informações de todas as consultas realizadas no ambulatório durante um período de 6 meses, de setembro de 2016 a março de 2017.

Os seguintes dados foram coletados: número de problemas da farmacoterapia e intervenções realizadas em cada consulta, comorbidades e seu estado situacional. $\mathrm{O}$ estado situacional das condições clínicas foi classificado pelo profissional que realizou a consulta de acordo com o enquadramento em categorias previamente definidas no serviço: CON (controlada), NCO (não controlada), SAD (sob avaliação diagnóstica), DES (desconhecido), MPA (melhora parcial), PPA (piora parcial) e CUR (cura). Esta classificação foi realizada de acordo com a anamnese direcionada a condição clínica envolvida, exames laboratoriais e clínicos, quando pertinentes, solicitados pelo farmacêutico ou outros profissionais de saúde que acompanharam o paciente no período ${ }^{24}$.

$\mathrm{O}$ estudo foi conduzido de acordo com as normas regulatórias de pesquisa com seres humanos (Resolução 466/12 do CNS e complementares), e foi aprovado pelo Comitê de Ética em Pesquisa com Seres Humanos do HC/UFPR (CAAE: 17675013.0.0000.0096).
Para a análise estatística foi determinada a normalidade da distribuição dos parâmetros "comorbidades", "problemas da farmacoterapia” e "intervenções farmacêuticas", por meio do teste de Komolgorov-Smirnov. Considerando que as variáveis apresentaram normalidade, os resultados foram expressos em média e desvio padrão. Adicionalmente, o teste $\mathrm{T}$ foi utilizado para análise pareada entre as consultas. Todas as análises foram feitas no software SPSS para Windows versão 20.0 e Microsoft Excel 2010.

\section{RESULTADOS}

Foram coletados os dados de 116 pacientes. Destes pacientes, 60 $(51,72 \%)$ tiveram apenas uma consulta durante o período da coleta de dados, sendo que 15 destes (25\%) já vinham sendo previamente acompanhados e receberam alta nesta consulta. Com relação aos outros 56 pacientes, 30 (25,86\%) foram acompanhados em duas consultas, $19(16,38 \%)$ em três consultas, 4 (3,45\%) em quatro consultas e $3(2,59 \%)$ em cinco. Na tabela 1 encontra-se a quantidade em média de comorbidades apresentadas em cada estado situacional nas três primeiras consultas.

Conforme representado na tabela, os dados comparados demonstram um aumento significativo das comorbidades "controladas", bem como uma redução das "não controladas" entre a primeira e a segunda consulta. Isso corrobora com o esperado, considerando-se que dentre as intervenções realizadas incluíam-se orientações sobre a terapia medicamentosa, solicitação de exames laboratoriais, encaminhamento a consultas com outros profissionais da saúde e manejo de doses. Na terceira consulta houve uma tendência de redução de comorbidades não controladas juntamente com um aumento da melhora parcial, porém possivelmente devido à redução drástica do número amostral, esses resultados não alcançaram significância estatística.

A comorbidade mais prevalente foi a hipertensão arterial sistêmica, presente em $94,8 \%$ dos pacientes, seguida da doença arterial coronariana, presente em $69,8 \%$, e dislipidemia em $62,9 \%$ dos pacientes. Outras comorbidades apresentadas foram: diabetes (41,1\%), insuficiência cardíaca $(26,7 \%)$ e problemas estomacais (22,4\%). A figura 1 ilustra a correlação entre as comorbidades e o estado situacional na primeira consulta farmacêutica.

Observa-se que, para as três principais comorbidades apresentadas na primeira consulta, menos de $70 \%$ dos pacientes apresentavamse em situação controlada. A hipertensão arterial sistêmica foi a comorbidade que apareceu mais frequentemente não controlada, com 29,1\% dos pacientes neste estado situacional. Dentre os pacientes que apresentaram dislipidemia, além de apenas 50,7\% apresentarem a comorbidade controlada na primeira consulta, 20,5\% apresentavam esta situação desconhecida, ou seja, onde não havia diagnóstico confirmado pelo médico, ou faltavam resultados dos exames laboratoriais para sua avaliação.

Os dados relacionados a estas comorbidades na segunda consulta estão descritos na figura 2, no qual é possível perceber que houve um aumento no controle destas comorbidades.

Apesar da quantidade de pacientes hipertensos em situação controlada não ter aumentado, 8,9\% destes tiveram uma melhora parcial condição, reduzindo a quantidade de não controlados. $\mathrm{O}$ número de pacientes com doença arterial coronariana e dislipidemia controladas aumentou visivelmente, de $64,2 \%$ para $78,9 \%$ e de $50,7 \%$ para $75,7 \%$, respectivamente. Além disso, a quantidade de pacientes com dislipidemia em situação desconhecida diminuiu para $8,1 \%$.

O número médio de problemas da farmacoterapia foi $2,14( \pm 1,571)$ na primeira consulta, $1,93( \pm 1,661)$ na segunda e $1,77( \pm 1,883)$ na terceira consulta. Paralelamente, foram realizadas 3,03 $( \pm 1,803)$ intervenções na primeira consulta, $3,04( \pm 1,858)$ na segunda e $3,19( \pm 2,020)$ na terceira consulta.

Considerando a o número de problemas e intervenções farmacêuticas entre a primeira e segunda consulta, não houve diferença significativa em análise pareada. 
Tabela 1 - Estado situacional das comorbidades apresentadas pelos pacientes durante as consultas no período de setembro de 2016 a março de 2017

Primeira consulta $^{*}$ Segunda consulta $^{*}$ Terceira consulta $^{*} \quad{\text { Comparação } 1^{\mathrm{a}} \mathrm{e} 2^{\mathrm{a}}}^{\text {Comparação } 1^{\mathrm{a}} \mathrm{e} 3^{\mathrm{a}}}$

Número de comorbidades controladas

$\begin{array}{ccc}2,52 \pm 1,465 & 2,95 \pm 1,519 & 2,69 \pm 1,543 \\ 1,66 \pm 1,554 & 1,32 \pm 1,428 & 1,12 \pm 1,633 \\ 0,29 \pm 0,619 & 0,3 \pm 0,570 & 0,35 \pm 1,018 \\ 0,5 \pm 0,839 & 0,29 \pm 0,653 & 0,27 \pm 0,553 \\ 0,2 \pm 0,479 & 0,32 \pm 0,606 & 0,54 \pm 0,761 \\ 0,08 \pm 0,420 & 0,09 \pm 0,438 & 0,08 \pm 0,272 \\ 0,03 \pm 0,293 & 0,02 \pm 0,134 & 0,04 \pm 0,196\end{array}$

consulta - valor de $\mathrm{p}^{* *}$ consulta - valor de $\mathrm{p}^{* *}$

Número de comorbidades não controladas

Número de comorbidades sob avaliação diagnóstica

Número de comorbidades em estado desconhecido

Número de comorbidades em melhora parcial

Número de comorbidades em piora parcial

Número de comorbidades curadas

$0,02 \pm 0,134$

$\begin{array}{ll}<0,001 & >0,05 \\ 0,002 & >0,05 \\ >0,05 & >0,05 \\ >0,05 & >0,05 \\ >0,05 & >0,05 \\ >0,05 & >0,05 \\ >0,05 & >0,05\end{array}$

*Dados expressos em média \pm desvio padrão; ${ }^{* *}$ Teste de T-Student pareado

Figura 1 - Estado situacional das comorbidades na primeira consulta

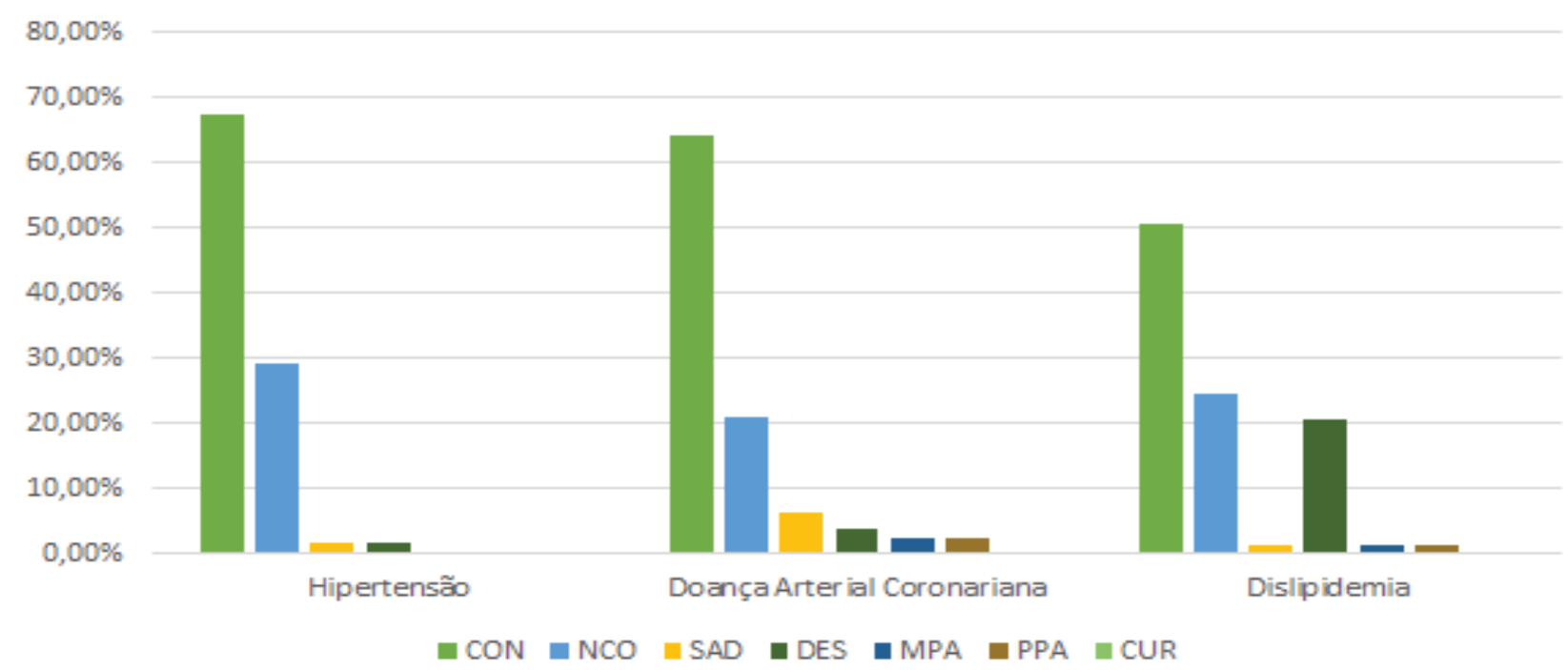

Legenda: $\mathrm{CON}=$ Comorbidades controladas; $\mathrm{NCO}=$ não controlado; $\mathrm{DES}=$ desconhecido; $\mathrm{MPA}=$ melhora parcial; $\mathrm{PPA}=$ piora parcial; $\mathrm{CUR}=$ curado

Figura 2 - Estado situacional das comorbidades na segunda consulta

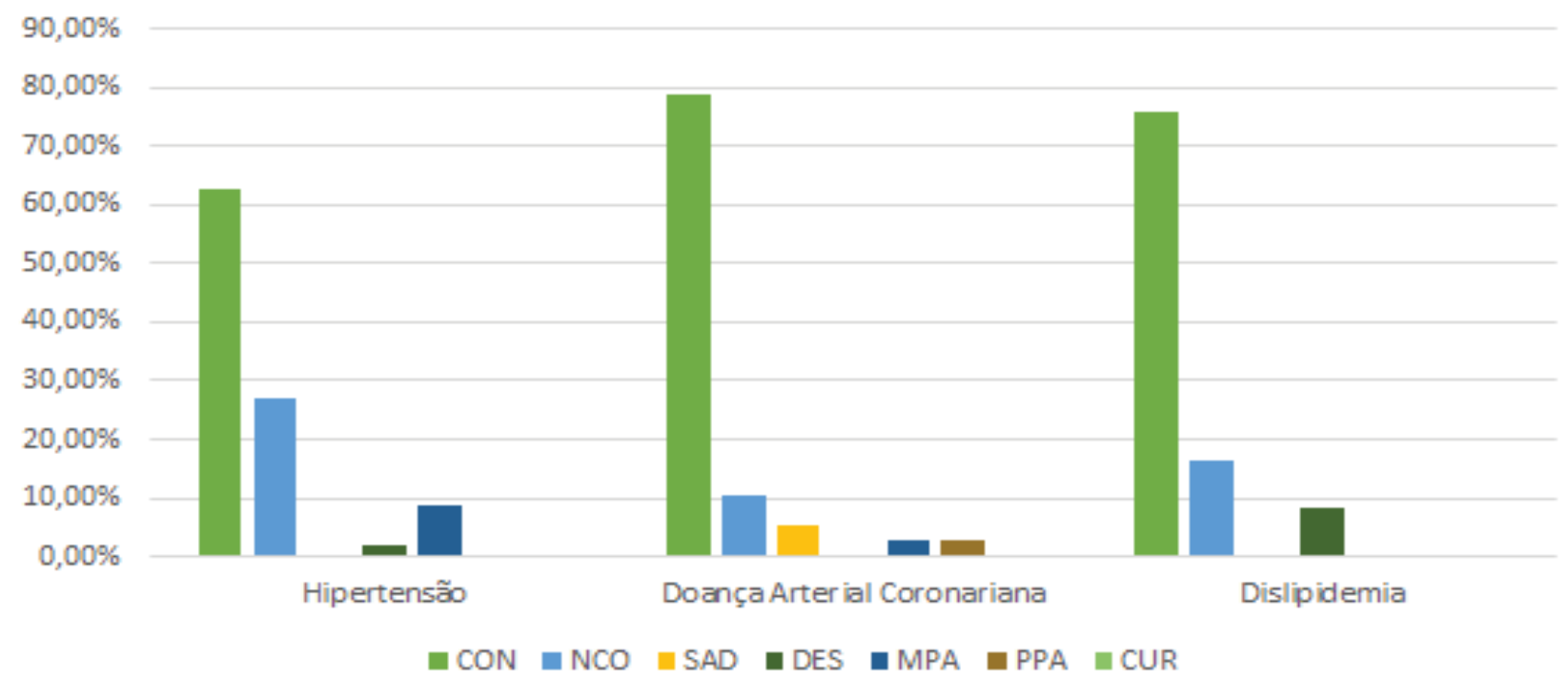

Legenda: $\mathrm{CON}$ = Comorbidades controladas; $\mathrm{NCO}$ = não controlado; $\mathrm{DES}=$ desconhecido; $\mathrm{MPA}=$ melhora parcial; $\mathrm{PPA}=$ piora parcial; $\mathrm{CUR}=$ curado 
Tabela 2 - Comparação pareada do número problemas e intervenções entre a primeira e a segunda consulta

\begin{tabular}{lccc}
\hline & Consulta 1 & Consulta 2 & $\begin{array}{c}\text { Valor } \\
\text { de "p" }\end{array}$ \\
\hline $\begin{array}{l}\text { Número de Problemas da } \\
\text { farmacoterapia }\end{array}$ & $2,14( \pm 1,571)$ & $1,93( \pm 1,661)$ & $>0,05$ \\
Número de intervenções & $3,03( \pm 1,803)$ & $3,04( \pm 1,858)$ & $>0,05$
\end{tabular}

${ }^{*}$ Dados expressos em média \pm desvio padrão

\section{DISCUSSÃO}

Este estudo demonstrou que as comorbidades mais incidentes nos pacientes analisados foram de doenças crônicas, que exigem um cuidado contínuo, a partir do uso de uma terapia farmacológica e/ou mudanças de hábitos de vida. A hipertensão arterial sistêmica e dislipidemia, condições mais prevalentemente encontradas na população do estudo, são consideradas fatores risco cardiovascular independentes, e podem gerar desfechos clínicos importantes, como a doença arterial coronariana e outros problemas de saúde, como doenças vasculares periféricas e doença renal crônica ${ }^{25}$.

As doenças crônicas não transmissíveis são doenças causadas por vários fatores que podem ou não ser evitadas e atualmente constituem a principal causa de mortalidade no Brasil e no restante do mundo. De acordo com dados do Sistema de Informações sobre Mortalidade (SIM), 72,6\% de todas as mortes ocorridas no país em 2013 foram causadas por alguma $\mathrm{DCNT}^{24}$. As doenças crônicas não transmissíveis que mais causam mortes no mundo são doenças cardiovasculares, respiratórias crônicas, cânceres e diabetes, doenças que poderiam ser prevenidas ou controladas de forma efetiva pela melhoria do atendimento dos sistemas de saúde às necessidades destes pacientes ${ }^{26}$.

Outro dado relevante apontado nesta investigação foi a constância da média de problemas e a necessidade recorrente de intervenções farmacêuticas. Isso indica, de maneira pouco surpreendente, que uma única consulta não é o suficiente para identificar e resolver todos os problemas apresentados. Alia-se a isso as possíveis mudanças no quadro clínico do paciente e o surgimento de novos problemas entre uma consulta e outra.

O acompanhamento clínico do farmacêutico já foi comprovado como sendo de extrema importância para a obtenção de desfechos positivos na saúde do paciente, como o uso de uma terapia mais segura, melhor adesão ao tratamento e melhoria na qualidade de vida ${ }^{27,28}$. Utilizando seus conhecimentos sobre os medicamentos, seus efeitos adversos, possíveis interações e modo de uso, o farmacêutico é capaz de avaliar a farmacoterapia do paciente e verificar a necessidade de realizar alguma intervenção para melhorar sua eficácia e reduzir ao máximo seus riscos ${ }^{29}$.

De maneira inovadora, o presente trabalho, através de uma abordagem integral das condições de saúde do paciente, indicou aumento significativo das comorbidades controladas entre a primeira e a segunda consulta, refletindo a importância das intervenções farmacêuticas para a melhora da condição de saúde global do paciente. Vale menção que diferença significativa foi observada já em curto prazo.

Em estudo retrospectivo realizado em hospital de alta complexidade publicado em 2008, Nunes e colaboradores ${ }^{29}$ analisaram estatisticamente as intervenções farmacêuticas realizadas e comprovaram sua eficácia na detecção e prevenção de eventos adversos causadas pela farmacoterapia, reafirmando a importância da inclusão do profissional farmacêutico na equipe multiprofissional de saúde.

Em consonância com os dados apresentados, em um estudo conduzido nas áreas rurais do Alabama publicado em 2003, Taylor, Byrd e Krueger comprovaram que a implementação de um programa de educação em medicamentos, com o monitoramento dos problemas relacionados à terapia e a inspeção das prescrições pelo farmacêutico a partir de consultas, possibilitou uma redução dos erros de prescrição, bem como um melhor controle das doenças e uma melhora na qualidade de vida ${ }^{30}$.

Da mesma forma, Jaber e colaboradores (1996) realizaram um estudo randomizado controlado em pacientes portadores de diabetes para verificar os efeitos do cuidado farmacêutico sobre esta população específica. O grupo de pacientes que recebeu as orientações com relação à doença, os medicamentos, exercícios físicos, dieta e automonitoramento teve uma melhora significativa nos resultados de hemoglobina glicada e glucose plasmática quando comparados a pacientes que continuaram recebendo o atendimento médico padrão, demonstrando a efetividade das intervenções farmacêuticas na melhoria da qualidade de vida ${ }^{31}$.

Como limitações do presente estudo, destacam-se a falta de um grupo comparador e o pequeno tamanho amostral. Entretanto, visto a escassez de evidências nacional na área, acreditamos que o mesmo contribui para a formulação de hipóteses e investigação robusta através de outros desenhos metodológicos.

Em resumo, partindo dos resultados obtidos, é possível inferir uma tendência de melhora dos resultados em do paciente. Contudo, para reafirmar a importância das consultas farmacêuticas sobre o estado de saúde do paciente seria interessante a realização de estudos controlados randomizados.

\section{CONCLUSÕES}

A análise dos dados obtidos pelos prontuários das consultas farmacêuticas, considerando o estado de saúde global do paciente, realizadas no período de seis meses, demonstrou que as intervenções farmacêuticas contribuíram para o controle das comorbidades dos pacientes atendidos.

\section{Fontes de Financiamento}

O presente trabalho não recebeu financiamento para sua realização.

\section{Conflitos de Interesse}

Os autores declaram inexistência de conflito de interesse.

\section{Colaboradores}

Todos os autores contribuíram igualmente para a concepção e projeto, análise e interpretação dos dados; redação do artigo, revisão crítica relevante do conteúdo intelectual e aprovação final da versão a ser publicada. Dessa forma, todos se responsabilizam pelas informações do trabalho, garantindo exatidão e integridade de qualquer parte da obra.

\section{Agradecimentos}

Não há.

\section{REFERÊNCIAS}

1. 1.Conselho Federal de Farmácia. Serviços farmacêuticos diretamente destinados ao paciente, à família e à comunidade contextualização e arcabouço conceitual. Distrito Federal, Conselho Federal de Farmácia; 2016. Disponível em: http://www.cff.org.br/userfiles/Profar_ Arcabouco_TELA_FINAL.pdf, 7 de junho de 2017.

2. Gastelurrutia MA, Llimós FF, Delgado PG, et al. Barriers and facilitators to the dissemination and implementation of cognitive services in Spanish community pharmacies. Granada, Pharm Pract; 2005. Disponível em http://www.redalyc.org/resumen. oa?id=69030201,27 de novembro de 2014 .

3. Bisson, MP. Farmácia Clínica \& Atenção Farmacêutica, 3a edição. São Paulo: Manole; 2016.

4. American Society of Health-System Pharmacists. ASHP Statement on Pharmaceutical Care. 1993. Disponível em https://www. ashp.org/-/media/assets/policy-guidelines/docs/statements/ 
pharmaceutical-care ashx?la=en \&hash=15F6B2BA0CF81920A246 FED05797C043DFC39FDD, 30 de agosto de 2017.

5. Pereira LRL, Freitas O. A evolução da Atenção Farmacêutica e a perspectiva para o Brasil. Rev Bras Cienc Farm, 2008, 44 (4):601-12.

6. Ivama AM, Noblat L, Castro, MS, et al. Consenso brasileiro de atenção farmacêutica: proposta. Distrito Federal, Organização PanAmericana da Saúde; 2002. Disponível em http://bvsms.saude.gov. br/bvs/publicacoes/PropostaConsensoAtenfar.pdf, 1 de setembro de 2017.

7. Fraccaro P, Arguello CM, Ainsworth J, et al. Adoption of Clinical Decision Support in Multimorbidity: A Systematic Review. 2015 Jan 7.Disponível em http://www.ncbi.nlm.nih.gov/pubmed/25785897, 8 de janeiro de 2015.

8. Santschi V, Chiolero A, Paradis G, et al. Pharmacist interventions to improve cardiovascular disease risk factors in diabetes: a systematic review and meta-analysis of randomized controlled trials. American Diabetes Association; 2012 Dec 1. Disponível em http://www.ncbi. nlm.nih.gov/pubmed/23173140, 25 de setembro de 2017.

9. Rotta I, Salgado TM, Silva ML, et al. Effectiveness of clinical pharmacy services: an overview of systematic reviews (2000-2010). Springer Netherlands, International Journal of Clinical Pharmacy, 2015, 23;37(5):687-97

10. Pousinho S, Morgado M, Falcão A, et al. Pharmacist Interventions in the Management of Type 2 Diabetes Mellitus: A Systematic Review of Randomized Controlled Trials. J Manag Care Spec Pharm, 2016, 22(5): 493-515.

11. Antoine SL, Pieper D, Mathes T, et al. Improving the adherence of type 2 diabetes mellitus patients with pharmacy care: a systematic review of randomized controlled trials. BMC Endocr Disord, 2014, $14: 53$.

12. Lavan AH, Gallagher P. Predicting risk of adverse drug reactions in older adults. Cork, Irlanda, Ther Adv drug Saf, 2016, 7(1):11-22.

13. Cortejoso L, Dietz RA, Hofmann G, et al. Impact of pharmacist interventions in older patients: a prospective study in a tertiary hospital in Germany. Nuremberg, Alemanha, Clin Interv in Aging, 2016, 11:1343-50.

14. Chumney EC, Robinson LC. The effects of pharmacist interventions on patients with polypharmacy. Granada, Pharm Pract, 2006, 4(3):103-9.

15. Lee J, Alshehri S, Kutbi H, et al. Optimizing pharmacotherapy in elderly patients: the role of pharmacists. Integr Pharm Res Pract, 2015, 4:101.

16. Gupta P, Patel P, Štrauch B, et al. Risk Factors for Nonadherence to Antihypertensive Treatment. Hypertension, 2017, 69(6):1113-20.

17. Platt $\mathrm{AB}$, Localio $\mathrm{AR}$, Brensinger $\mathrm{CM}$, et al. Risk factors for nonadherence to warfarin: results from the IN-RANGE study. Pharmacoepidemiol Drug Saf, 2008, 17(9):853-60.

18. Vasconcelos AMN, Gomes MMF. Transição demográfica: a experiência brasileira. Distrito Federal, Epidemiol e Serviços Saúde, 2012, 21(4):539-48.

19. Miranda GMD, Mendes A da CG, Silva ALA da. Population aging in
Brazil: current and future social challenges and consequences. Rio de Janeiro, Rev Bras Geriatr e Gerontol, 2016, 19(3):507-19.

20. Vitkil KK, Blix HS, Moger TA et al. Polypharmacy as commonly defined is an indicator of limited value in the assessment of drugrelated problems. Br J Clin Pharmacol, 2006, 63(2):187-95.

21. Linjakumpu T, Hartikainen S, Klaukka T, et al. Use of medications and polypharmacy are increasing among elderly. Journal of Clinical Epidemiology, 2002, 55:809-17.

22. World Health Organization. Adherence to long-term therapies: evidence for action. World Health Organization; 2015. Disponível em: http://www.who.int/chp/knowledge/publications/adherence report/en/, 25 de setembro de 2017.

23. Brasil. Ministério da Saúde. Secretaria de Ciência, Tecnologia e Insumos Estratégicos. Resultados do projeto de implantação do cuidado farmacêutico no Município de Curitiba / Ministério da Saúde, Secretaria de Ciência, Tecnologia e Insumos Estratégicos, Departamento de Assistência Farmacêutica e Insumos Estratégicos. Distrito Federal, Ministerio da Saude, 2015, 200 p.

24. Brasil. Ministério da Saúde. Secretaria de Ciência, Tecnologia e Insumos Estratégicos. Departamento de Assistência Farmacêutica Insumos Estratégicos. Planejamento e implantação de serviços de cuidado farmacêutico na Atenção Básica à Saúde: a experiência de Curitiba / Ministério da Saúde, Secretaria de Ciência, Tecnologia e Insumos Estratégicos. Departamento de Assistência Farmacêutica e Insumos Estratégicos. Distrito Federal, Ministério da Saúde, 2014. 120 p.: il. - (Cuidado farmacêutico na atenção básica; caderno 3).

25. Brasil. Ministério da Saúde. Portal da Saúde. Disponível em http:// portalsaude.saude.gov.br/index.php/o-ministerio/principal/leiamais-o-ministerio/671-secretaria-svs/vigilancia-de-a-a-z/doencascronicas-nao-transmissiveis/14125-vigilancia-das-doencas-cronicasnao-transmissiveis, 27 de setembro de 2017;

26. Organização Mundial da Saúde. Global action plan for the prevention and control of noncommunicable diseases 2013-2020. Geneva, Suiça, World Health Organization. Disponível em: http://apps.who. int/iris/bitstream/10665/94384/1/9789241506236_eng.pdf, 28 de setembro de 2017.

27. Shah, A. Pharmacy Intervention in the Medication-use Process. Deen Hag, Holanda, 2009. Disponível em https://www.fip.org/files/fip/ Patient\%20Safety/PatientSafetyAdvidShah.pdf, 28 de setembro de 2017.

28. Sartore, ME, Ehman, KM, Good, CB. The Significance of Pharmacy Interventions: An Updated Review in the Presence of Eletronic Order Entry. The American Journal of Pharmacy, 2014, 6 (2): 24-30.

29. Nunes PHC, Pereira BMG, Nominato JCS, et al. Intervenção farmacêutica e prevenção de eventos adversos. Revista Brasileira de Ciências Farmacêuticas, 2008, 44(4):691-99.

30. Taylor, CT, Byrd, DC, Krueger, K. Improving primary care in rural Alabama with a pharmacy initiative. Am J Health-Syst Pharm, 2003, 60:1123-1129.

31. Jaber LA, Halapy H, Fernet M, et al. Evaluation of a pharmaceutical care model on diabetes management. Ann Pharmacother, 1996, $30(3): 238-243$. 